\title{
Green Constructing an Intelligent Temperature- Regulating Fabric with Multiple Heat-Transfer Capabilities
}

Bing Qi

Jiangnan University

Feiyu Wang

Jiangnan University

Qin Chen

Jiangnan University

Bo Xu

Jiangnan University

Ping Wang ( $\sim$ pwang@jiangnan.edu.cn )

Jiangnan University

Zhou Man

Jiangnan University

Yuanyuan Yu

Jiangnan University

Qiang Wang

Jiangnan University

\section{Research Article}

Keywords: Cotton fabric, hydroxylated boron nitride (BN-OH), poly ( $\mathrm{N}$-isopropyl acrylamide) (PNIPAM), horseradish peroxidase, temperature responsiveness

Posted Date: October 14th, 2021

DOl: https://doi.org/10.21203/rs.3.rs-946678/v1

License: (1) This work is licensed under a Creative Commons Attribution 4.0 International License.

Read Full License 


\section{Abstract}

Textiles with heat management function have good effects on improving human comfort during sport. However, it is still a great challenge to endow textiles with responsiveness to external environmental changes. Herein, we developed an intelligent temperature-regulating cotton textile with multiple heat transfer capability by a two-step method. Firstly, hydroxylated boron nitride (BN-OH) nanosheet dispersion liquid was prepared using a two-step ultrasonic-alkali treatment. Subsequently, enzymatic graft polymerization of N-isopropyl acrylamide (NIPAM) onto cotton fibers were performed using horseradish peroxidase (HRP). The composite cotton fabric, containing entrapped $\mathrm{BN}-\mathrm{OH}$ exhibits unique temperatureregulating ability, and the thermal diffusivities in vertical and parallel directions reach 1.2 and 1.7 times of the untreated, respectively. This can be attributed to the temperature responsiveness of poly-NIPAM (PNIPAM) and the increase in the packing density of the thermal conductive nanosheets at high temperatures. Meanwhile, the PNIPAM covering the fiber surfaces slowly expands at low temperatures, accordingly minishes the gap sizes between fabric yarns and endows the fabric with improved heat preservation effects. The present work provides a facile and green strategy for developing the intelligent textiles with ambient temperature self-response ability.

\section{Introduction}

Extreme sports like marathons have become a new fashion in the field of national fitness in recent years. However, some sports always take a long time under uncertain weather conditions, which poses a challenge to many runners. For example, the anaerobic metabolism of the human body will be intensified in a high-temperature environment, easily causing the accumulation of lactic acid. On the contrary, cold weather would reduce muscle ductility and many other discomforts (Rodrigues Júnior et al., 2020; Binkley et al., 2002). Thus, it is necessary to create a comfortable micro-environment for runners by wearing temperature-regulating textiles. Application performances of textiles can be easily adjusted by using special fibers and diverse finishing, for constructing functional fabrics and satisfying the increasing demands from customers (Bartkowiak et al., 2017; Yang et al., 2017; Couto et al., 2011; Cui et al., 2018). Comparatively, fabric finishing is an effective way to impart desired functionality to textiles. More recently, many finishing works were carried out for developing functional textiles with adjustable temperature and humidity performances, such as moisture-wicking (Wang et al., 2018), temperaturesensitive (Salaün et al., 2010), photothermal conversion (Cheng et al., 2020), and thermal insulation (Guo et al., 2021). Unfortunately, these textiles always exhibit a single moisture or heating capability, and it can hardly rapidly respond to the changes in external temperature and moisture, which limit their further practical applications to some extent.

More recently, the research work on room temperature phase change materials provides the possibility of intelligent temperature adjustment for clothing. Poly ( $\mathrm{N}$-isopropyl acrylamide) (PNIPAM), obtained from the polymerization of N-isopropyl acrylamide (NIPAM) is a kind of widely used temperature responsive polymer. It has a lower critical solution temperature (LCST) (around $32^{\circ} \mathrm{C}$ ) close to human body temperature, companying with good biocompatibility (Xia et al., 2013). It has the characteristics of 
shrinking at high temperature but swelling at low temperature through the changes in the water absorbing behaviors, and holds great promise to be used in many practical areas, including drug delivery (Sasidharan et al., 2016; You et al., 2008), surface wettability (Ye et al., 2015; Yu et al., 2012; Li et al., 2010), intelligent surface materials (Turan et al., 2010), and sensor analysis (Chen et al., 2013).

Accordingly, such polymer provides an alternative for the development of temperature-responsive textiles.

Boron nitride $(\mathrm{BN})$, commonly named white graphite is a promising thermal conductive material because of its inherent excellent electrical insulation properties and high thermal conductivity (J. Chen et al., 2017; Q. Li, Chen, et al., 2015; Q. Li, Zhang, et al., 2015). It has been widely applied in the fields of sensors (Lin \& Connell, 2012), catalysts (Sun et al., 2016), high-performance composite (Q. Li et al., 2014), and superhydrophobic coatings. To obtain a wearable cooling textile, Wu et al prepared a hydrophilic and thermally conductive regenerated cellulose multifilaments via adding BN nanosheets into cellulose solution followed by wet-spinning (Wu et al., 2019). However, for natural fibers, it is not easy to achieve the entrapment of such nanosheets into fiber interior because of the tight fibrous structure. Therefore, it is necessary to develop a simple and feasible finishing method for the preparation of temperatureadjustable textiles, via the combined use of thermal conductive and temperature-sensitive materials.

Herein, we proposed a facile and green strategy for constructing an intelligent temperature-regulating natural textile using PNIPAM and BN nanosheets. As shown in Fig. 1A, hydroxylated boron nitride (BN$\mathrm{OH}$ ) was prepared by a two-step ultrasonic-alkali treatment, and vinyl groups were introduced to cotton fibers for obtaining improved reactivity. Subsequently, horseradish peroxidase (HRP) catalyzed the graft polymerization of PNIPAM onto fiber surfaces via radical reaction (Zhou et al., 2017), aiming at constructing an interpenetrating 3D thermal network with entrapped $\mathrm{BN}-\mathrm{OH}$ nanosheets (Fig. 1B). At a low temperature, PNIPAM tends to swell and accordingly increases the gaps between $\mathrm{BN}-\mathrm{OH}$ nanosheets, endowing the cotton fabric with low thermal conductivity and improved heat preservation effects. On the contrary, a high temperature might initiate the shrinking of PNIPAM owing to its phase transition, which accordingly increases the packing density of the nanosheets covering cotton fibers and enlarges the gap size between the warp and weft yarns, resulting in the formation of heat-transfer paths with efficient heat conduction ability (Fig. 1C). This work provides a facile enzymatic approach for developing functional textiles to satisfy the complex requirements of human body temperature regulation in different environments.

\section{Materials And Methods}

\subsection{Materials}

Cotton fabric was provided by Lianfa Textile Co. (Jiangsu, China). N-isopropyl acrylamide (NIPAM, 98\%) and N,N'-Methylene bisacrylamide (MBA, 99\%) were purchased from Adamas (Shanghai, China). h-BN $(98.5 \%, 1-2 \mu \mathrm{m})$ was supplied by Yinuokai Technology Co. (Beijing, China). Horseradish peroxidase (HRP), with an activity of $300 \mathrm{U} / \mathrm{mg}$, was provided by Aladdin Reagent Co. (Shanghai, China). Sodium dodecyl sulfate (SDS) and other regents employed were of analytical grade. 


\subsection{Preparation of BN-OH nanosheets and vinylated cotton fabric}

Multilayer BN powder was firstly tip-type sonicated in SDS solution (1mol/L) and dispersed for $3 \mathrm{~h}$ (Shi et al., 2020). Then $5 \mathrm{~mol} / \mathrm{L}$ sodium hydroxide was added to the resulting $\mathrm{BN}$ dispersion and transferred to a three-necked flask for hydroxylation (Lu et al., 2021). After refluxing at $120^{\circ} \mathrm{C}$ for $18 \mathrm{~h}$, the obtained mixture solution was neutralized with hydrochloric acid, and further dialyzed against deionized water for 1 week to remove the residual salts. Finally, the $\mathrm{BN}-\mathrm{OH}$ nanosheets were obtained after drying at $60^{\circ} \mathrm{C}$.

To improve the cotton reactivity, scoured cotton fabric was immersed in $50 \mathrm{~g} / \mathrm{L}$ of methacrylic anhydride for vinylation, and the reaction was conducted at $4^{\circ} \mathrm{C}$ and approximately $\mathrm{pH} 7-8$ for $24 \mathrm{~h}$ (Kang et al., 2017). Afterward, the vinylated cotton fabric was thoroughly rinsed with ethanol, then air-dried.

\subsection{Preparation of thermal conductive fabric with temperature-regulating performance}

Thermal conductive cotton fabric was enzymatically prepared via HRP-catalyzed graft polymerization of PNIPAM. Briefly, the vinylated cotton fabric was immersed into a mixture solution containing $1 \mathrm{~g}$ of NIPAM, $0.05 \mathrm{~g} \mathrm{MBA}, 3 \mathrm{mg} \mathrm{HRP}, 200 \mu \mathrm{L}$ acetylacetone, $40 \mu \mathrm{L} \mathrm{H}_{2} \mathrm{O}_{2}$, and $20 \mathrm{~mL}$ of phosphate buffer ( $\mathrm{pH}=$ 7) (Y. Yang et al., 2021). A certain amount of $\mathrm{BN}-\mathrm{OH}$ was selectively added into the incubation bath for regulating the thermal conductive performance of cotton fabric. After the free-radical polymerization was performed at $37^{\circ} \mathrm{C}$ for $24 \mathrm{~h}$, the temperature-regulating cotton fabric was obtained, then washed and airdried. Eventually, a thin layer of PNIPAM covering the yarn surfaces of cotton fabric, with and without entrapped $\mathrm{BN}-\mathrm{OH}$ was obtained.

\subsection{Characterizations}

\subsubsection{BN-OH structure and temperature sensitivity of PNIPAM}

Morphology of the $\mathrm{BN}-\mathrm{OH}$ nanosheets was examined by a $\mathrm{H}-7000$ transmission electron microscopy (TEM) (Hitachi, Japan). Fourier transform infrared spectroscopy (FTIR) was used to analyze the characteristic structure of $\mathrm{BN}$ with different treatments, the absorbance in the wavelength range from 500 to $4000 \mathrm{~cm}^{-1}$ was recorded at a resolution of $4 \mathrm{~cm}^{-1}$. The particle size and particle size distribution of BN were examined by zeta potential and particle size analyzer (Brookhaven Instruments, USA). The thermal responsive behaviors of PNIPAM, ranged from 22 to $42^{\circ} \mathrm{C}$ were investigated by determining the changes in the absorbance of PNIPAM solutions at $500 \mathrm{~nm}$ using a UV-1800 spectrophotometer (Shimadzu, Japan).

\subsubsection{Structural and morphological characterization of cotton}


The introduced vinyl groups on cotton fabric was identified by an iodine-mediated chromogenic reaction. In a typical experiment, iodine solution was firstly prepared according to the following reaction: $\mathrm{IO}^{3-}+5 \mathrm{I}^{-}+6 \mathrm{H}^{+}=3 \mathrm{I}_{2}+3 \mathrm{H}_{2} \mathrm{O}$. Then, the modified fabric was immersed in the iodine solution for $5 \mathrm{~min}$, followed by storing in darkness. The change in the color appearance of the fabric surface was used to ensure whether the vinyl group from methacrylic anhydride was successfully coupled onto fiber surfaces or not. Meanwhile, FTIR was also used to identify the changes before and after vinylation modification. Surface morphologies of cotton fabric samples, based on different treatments were observed using an SU1510 scanning electron microscope (HITACHI, Japan).

\subsubsection{Thermal diffusivity}

The out-of-plane and in-plane thermal diffusivity of fabric were measured from 20 to $45^{\circ} \mathrm{C}$ with an LFA 457 analyzer (NETZSCH, Germany). The laser source emits a light pulse in an instant and uniform way towards the lower surface of the fabric sample, and the surface layer absorbs light energy, transforming the light into heat. Therefore, the temperature rises instantaneously and acts as the hot end to transfer energy to the cold end (upper surface or around) in one-dimensional heat spread. The rise of the temperature of the corresponding part was continuously measured using an infrared detector and the thermal diffusivity was calculated as follows:

$$
\alpha=0.1388 \times d^{2} \div t_{50}
$$

$\boldsymbol{a}$ Thermal diffusivity $\mathrm{mm}^{2} / \mathrm{s}$

$d^{2}$ Thickness of sample

$t_{50}$ The half heating time, which is the time required for the upper surface temperature of the sample to rise to half of the maximum value after receiving the light pulse irradiation.

\subsubsection{Thermal conductivity and insulation performance}

Cotton fabrics were rolled into columns and sealed at both ends, then placed into an oven at $55^{\circ} \mathrm{C}$ and a refrigerator at $4^{\circ} \mathrm{C}$, respectively. Internal temperature of each sample was monitored and recorded by a temperature probe connected to inside of the prepared fabric sample. Thermal images for the fabric samples were taken by a TiS55 thermal imager (Fluke, USA), with the working distance of approximately $30 \mathrm{~cm}$ between the camera and fabric surface.

\subsubsection{Wearable performances of cotton fabrics}

The anti-ultraviolet performance of each cotton fabric was tested using a YG(B)912E ultraviolet transmission analyzer (Darong Co., China), expressed as UV protective factor (UPF). Wettability of fabric sample was determined by a JC2000D4 contact angle goniometer (Zhongchen Digital Technology Co., China), meanwhile, it was also represented by the height of wetting in the vertical direction of fabric 
surface. Furthermore, air permeability was also evaluated measured by an automated YG461E permeability instrument (Ningbo Textile Instrument Co., China), respectively.

\section{Results And Discussion}

\subsection{Effects of ultrasonic-alkali treatment on morphology and structure of $\mathrm{BN}-\mathrm{OH}$ nanosheets}

$\mathrm{BN}-\mathrm{OH}$ nanosheets were prepared through the combined use of ultrasound and alkali treatments, aiming at constructing continuous thermal paths onto to the target substrate of cotton surface. As shown in Fig. $2 \mathrm{~A}_{1}$, the few-layered $\mathrm{BN}-\mathrm{OH}$ nanosheet can be observed due to its ultimately thin shapes. Meanwhile, some of the stacked $\mathrm{BN}-\mathrm{OHs}$ with single or a few layers in a small size were also detected, exhibiting a lateral size of the aggregation state less than $350 \mathrm{~nm}$ (Fig. 2A $\mathbf{A}_{2}$ ) (Tian et al., 2019). Stability of the BN suspensions treated with ultrasound and alkali were also examined, and the observed appearances after storing for different days are presented in Fig. 2B. Both of the pristine dispersion and the ultrasoundtreated exhibited stratified appearance after standing for 3 days, while the $\mathrm{BN}-\mathrm{OH}$ nanosheets dispersion with ultrasound-alkali process kept uniform appearances within 3 days and slightly stratified until 5 days. Figure $2 \mathrm{C}$ illustrates the particle sizes of the above-mentioned three $\mathrm{BN}$ suspensions, which are mainly distributed in the ranges of $400-1900 \mathrm{~nm}, 200-700 \mathrm{~nm}$, and $200-700 \mathrm{~nm}$, respectively. The result is in good agreement with that in Fig. 2B, supporting the effective exfoliation of BN during the treatment (Zhu et al., 2014).

Characteristic structures of different BN samples are depicted in Fig. 2D, two strong FTIR bands at approximately $1343 \mathrm{~cm}^{-1}$ and $807 \mathrm{~cm}^{-1}$ are observed for the three samples, mainly attributed to the inplane stretching and the out-of-plane bending mode of B-N (Xiao et al., 2015). Furthermore, a characteristic peak at $3440 \mathrm{~cm}^{-1}$ appears in the spectra of BN treated with ultrasound-alkali process, revealing the successful introduction of hydroxyl groups into the nanosheets. According to the results in Fig. 2, it can be inferred that ultrasound mainly peels off BN to form nanosheets, while alkali treatment might introduce more hydrophilic hydroxyl groups exposed on the nanosheet surfaces.

It is well-known that BN has good thermal conductivity, thus it can be used to endow the BN-based composites with improved thermal conductivity. In our experiment, optical and infrared test systems were constructed to examine the thermal properties of the prepared $\mathrm{BN}-\mathrm{OH}$ nanosheets. As the model of textiles, the filter paper with and without BN were used, the surface temperature of each was recorded every $5 \mathrm{~s}$ upon heating at $40^{\circ} \mathrm{C}$, and the results are shown in Figure $\mathrm{S} 1$. When the filter paper was directly placed on the graphite heating plate, energy from the heating plate might be absorbed, which is similar to that the body continuously transfers a certain amount of heat from skin surface to the clothing. It can be seen that the surface temperature of filter paper containing BN-OH was a bit higher than that of the neat filter paper (about $0.7^{\circ} \mathrm{C}$ ) no matter how long it was placed, indicating that $\mathrm{BN}-\mathrm{OH}$ achieved a good heat transfer effect. To further investigate the effect of $\mathrm{BN}-\mathrm{OH}$ concentration on the thermal conductivity, an 
experimental device was constructed (Fig. 6A) (Y. Yang et al., 2021), and the change in the internal temperature of the fabric was monitored by using the probe to evaluate the thermal conductivity of BN$\mathrm{OH}$. The results in Figure $\mathrm{S} 2$ reveals that the fabric immersed in $10 \mathrm{~g} / \mathrm{L}$ of $\mathrm{BN}-\mathrm{OH}$ nanosheets displays the highest internal temperature and the best thermal conductivity. This can be explained as that the heat conduction paths can hardly be efficiently formed with low content of $\mathrm{BN}-\mathrm{OH}$, owing to the lack of close overlaps between the nanosheets. In contrast, excessive dosage of $\mathrm{BN}-\mathrm{OH}$ may block the small gaps between warp and weft yarns, which will lead to unacceptable blocking of heat convection. Thus, $10 \mathrm{~g} / \mathrm{L}$ $\mathrm{BN}-\mathrm{OH}$ as the optimal dosage was selected for the subsequent processing.

\subsection{Morphology and structure of the prepared temperature- regulating cotton fabric}

To investigate the thermal response of PNIPAM, the transmittances of the polymer aqueous solutions were measured at different temperatures. Figure $3 \mathrm{~A}$ reveals that the transmittance of the polymer aqueous solution starts to decrease at $30^{\circ} \mathrm{C}$ and reaches the minimum at $32^{\circ} \mathrm{C}$. Considering that the LCST of a thermo-responsive polymer reflects the temperature at which the transmittance of an aqueous polymer solution is $50 \%$,(Ma et al., 2010) the PNIPAM solution exhibited a transparent appearance at temperatures lower than $32^{\circ} \mathrm{C}$, then turned to be milky white at a temperature higher than LCST. Figure 3B shows the change in the sample length of PNIPAM hydrogel, it shrinks from 3.5 to $2.2 \mathrm{~cm}$ when the temperature rises from room temperature up to $50^{\circ} \mathrm{C}$. Figure $3 \mathrm{C}$ shows the structure and temperature response mechanism of PNIPAM, PNIPAM shows a stretched coil structure at low temperature, due to the hydrogen bonds and van der Waals forces from the hydrophilic interaction of amide bonds of PNIPAM and water molecules. With the increase of temperature, the polymer formed a compact colloidal structure owning to the entropy of the polymer system, and the hydrophobic interactions between molecules both increased.

The photographs for the fabric samples, colored with iodine solution are shown in Fig. 4A. After placing the treated sample in dark for 20 min, the color of the vinylaed cotton significantly faded, while the color of the untreated did not change. Structural characteristics of the cotton fabric were analyzed by FTIR, and the vinylated cotton fabric shows the peak at $1750 \mathrm{~cm}^{-1}$ corresponding to the $\mathrm{C}=\mathrm{O}$ stretching vibration, indicating that vinyl groups were successfully grafted onto cotton fibers through the esterification with anhydride. The introduction of double bonds onto the fiber surfaces were also verified by iodine-mediated chromogenic reaction. The infrared spectrum for the sample of Cotton-NIPAM is shown in Fig. 4B, the new strong peaks appear at $1640 \mathrm{~cm}^{-1}$ and $1545 \mathrm{~cm}^{-1}$ corresponds the stretching vibration of the amide bond in PNIPAM, verifying the formation of the phase-change polymer.

The intelligent temperature-regulating cotton fabric, containing the phase-change material of PNIPAM and thermal conductive $\mathrm{BN}-\mathrm{OH}$ nanosheets was prepared, and the surface morphology together with comparison samples are shown in Fig. 4C. The cotton fiber without any treatments exhibits a smooth surface, while some flaky fragments distributed on the surface of the fiber was observed for the sample of Cotton-BN-OH. After enzymatic graft polymerization of PNIPAM onto the vinylated cotton, a thin film of 
sediment scattered on the fiber surfaces. For the fabric based on the combined use of $\mathrm{BN}-\mathrm{OH}$ and PNIPAM, the fiber surfaces appeared the composite morphology of BN-OH wrapped by PNIPAM at room temperature, companying with moderate porosity inside of fabric yarns.

Air permeability of the cotton fabrics with different treatments were also examined, and the results are shown in Fig. 4D. For the samples of the untreated and cotton-BN-OH, the air permeability reached 202.16 and $186.12 \mathrm{~mm} / \mathrm{s}$, respectively. Meanwhile, no obvious changes in air permeability were detected under the two different temperatures of $40^{\circ} \mathrm{C}$ and $25^{\circ} \mathrm{C}$. After graft polymerization of PNIPAM, air permeability of the fabric sample was slightly reduced compared that of the untreated, mainly owing to the reduced porosity in the composite fabric. Moreover, as shown in Movie S1, a large amount of white smoke emerges from the glass bottle containing $\mathrm{HCl}$ covered with cotton-PNIPAM-BN-OH fabric, illustrating that the air permeability of the composite fabric is still maintained at an acceptable level. Meanwhile, the air permeability at high temperature was remarkably higher than that at low temperature. For better understanding the synergistic heat transfer mechanism of BN-OH and PNIPAM, Fig. 4D depicted the mechanism on the above phenomenon. At low temperatures, PNIPAM slowly swells after absorbing water molecules, which accordingly reduces the gap between yarns and to improve the heat insulation effect of the fabric. On the contrary, PNIPAM might shrink at high temperatures, which enlarges the gap sizes between fibers and yarns as well, endowing fabric with improved thermal convection in the vertical direction. The proposed mechanism meets the complex requirements of human body temperature regulation in different environments.

\subsection{Thermal conductivity behaviors of the prepared temperature-regulating cotton fabric}

Among the three forms of heat transfer pathways (i.e., conduction, convection, and radiation) of thermally regulating textiles, heat conduction plays an important role. When the heat is transferred by conduction, the energy is dissipated outward from the human body through clothing to the outside environment (Gao et al., 2017). Figures $5 \mathrm{~A}_{1}$ and $\mathbf{5} \mathrm{B}_{1}$ illustrate the schematic of the thermal conductivity measurement. The determined thermal diffusivities for different cotton fabrics are shown in Figs. $\mathbf{5 A}_{\mathbf{2}}$ and $\mathbf{5 B}_{2}$. It can be seen that both BN and PNIPAM might increase the thermal diffusivity of cotton fabrics to some extent, regardless of in vertical and parallel directions. For the sample combinedly treated with PNIPAM and $\mathrm{BN}-\mathrm{OH}$, when the ambient temperature was at $40^{\circ} \mathrm{C}$, the thermal diffusivities for the sample reaches 1.7 and 1.3 times in vertical and parallel directions, respectively, mainly owing to the synergistic effects of the PNIPAM and BN-OH nanosheets. PNIPAM on the fiber surfaces tended to shrink because of its temperature response, resulting in the formation of heat conduction path and the improvement of the thermal diffusion coefficient accordingly (Fig. $5 \mathrm{~A}_{3}, \mathbf{5 B}_{3}$ ). The combined use of PNIPAM and the BN-OH have a synergistic effect in promoting the heat conduction effect of the cotton fabric, realizing the switch between heat conduction and preservation at different temperatures.

The thermal conductivity behaviors of the composite cotton fabrics were also examined by detecting the dynamic temperature changes during heating and cooling. As shown in Fig. $6 \mathrm{~A}$, the treated cotton fabric 
was placed in an oven and a refrigerator, respectively, and the temperatures inside the wrap composite fabrics were recorded in real time by a probe. As can be seen Fig. $6 \mathrm{~B}$, when the fabric samples were placed at $55^{\circ} \mathrm{C}$, the cotton fabric containing PNIPAM and $\mathrm{BN}-\mathrm{OH}$ exhibits remarkably higher heat transfer efficacy than others except for cotton-BN-OH. While storing at $4^{\circ} \mathrm{C}$, the temperature inside the center of the composite fabric rose more slowly (Fig. 6C), revealing the certain heat preservation of the fabric at low temperatures.

The differences in thermal conductive performances for the cotton fabrics were further explained in Fig. 6D. For the composite cotton fabric, the NIPAM polymer covering fiber surfaces swells at low temperatures and accordingly reduces the air permeability, which endows the fabric with better insulation effects. Comparatively, high temperature leads to a shrinkage of phase-change material and improved air permeability, companying with the formations of thermal conductive path with overlapping nanosheets.

\subsection{Application performances of the temperature-regulating cotton fabric}

For the composite cotton fabric, the NIPAM polymer covering fiber surfaces swells at low temperatures and accordingly reduces the air permeability, which endows the fabric with better insulation effects. Comparatively, high temperature leads to a shrinkage of phase-change material and improved air permeability, companying with the formations of thermal conductive path with overlapping nanosheets.

Static cooling effects of the prepared cotton textiles in actual application were examined according to the reported methods (Gao et al., 2017). Cotton fabrics with different treatments were placed onto the back of hand, respectively, then the temperature distribution of each sample was recorded by an infrared camera (Fig. 7A). The results indicated that the outer surface temperature of cotton-PNIPAM-BN was a bit higher than that of others, which is close to the normal temperature of human skin (Fig. 7B), revealing that effective cooling effect was achieved. Similar results are depicted in Fig. 7C, the sample of cottonPNIPAM-BN-OH has more encouraging dynamic cooling effect than the other samples. Therefore, the heat generated by the human body can be effectively transferred via using cotton-PNIPAM-BN-OH fabric.

Other wearability performances for the composite cotton fabric were also concerned. Figure 8 shows the results of UV resistance ability, whiteness, wettability, and mechanical behavior. The combined use of BN$\mathrm{OH}$ and PNIPAM imparted an excellent protective effect to the composite fabric, and the UPF reaches approximately 53. Meanwhile, introduction of PNIPMA and BN-OH did less impacts to the color appearance to the cotton fabrics, and all of the measured Hunter whiteness were at approximately $83 \%$.

Wettability of fabric samples were measured, and expressed as wetting height and the static water contact angle (WCA) at room temperature. For the composite fabric of cotton-PNIPAM-BN-OH, although a slight decrease in the wettability is observed in Fig. $8 \mathrm{C}$, the wetting height still meets the basic requirement of textile wettability (approximately $8 \mathrm{~cm}$ ). The photograph in Fig. 8D shows the WCA of different samples, the water droplet on the BN-cotton fabric maintained a spherical shape for less than 1 $s$ before collapsing, and the spherical shape of the droplet collapsed altogether within $3 \mathrm{~s}$ on the PNIPAM- 
cotton fabric, and maintained a spherical shape for $4 \mathrm{~s}$ after contacting with cotton-PNIPAM-BN-OH fabric. The slight decrease in the hydrophilicity can be explained that the amide bond in the PNIPAM structure is able to combine with water molecules to form intermolecular hydrogen bonds at low temperatures, and the ability of amide bonds to bind water is weaker than that of hydroxyl groups. When the cotton fabric is modified, the hydroxyl groups of the internal fiber structure cannot directly contact the outside because of the tight arrangement of PNIPAM chains. Water molecules are required to pass through the PNIPAM layer to enter the fiber. However, thanks to the tight structure and the relatively weak ability of the amide bond to bind water, the process of water molecules entering the interior of the modified cotton fabric is relatively slow. (Bai et al., 2019) The results in Figs. 8E reveal that introduction of $\mathrm{BN}-\mathrm{OH}$ and PNIPAM also increases the bending rigidity of the fabric to some extent, mainly owing to the extra component phase-change polymer and nanosheets.

\section{Conclusion}

An intelligent temperature-regulating textile with excellent thermal management was successfully prepared, via the horseradish peroxidase-catalyzed graft polymerization of PNIPAM and BN-OH entrapment onto cotton fabrics. At high temperatures, the thermal diffusivity in the vertical direction of fabric reached 1.6 times compared to the untreated, exhibiting a surface temperature nearly $1.2^{\circ} \mathrm{C}$ higher than the latter. Relatively speaking, the polymer of PNIPAM on the fiber surfaces tended to can be restored to its original larger morphology at a low temperature, and the density of $\mathrm{BN}-\mathrm{OH}$ distributed in the polymer was reduced, thus playing a role in the heat protection of the human body by increasing thermal resistance. Meanwhile, $\mathrm{BN}-\mathrm{OH}$ has a certain degree of anti-ultraviolet performance (UPF > 50), which makes it have a better experience in outdoor activities. This work provides an effective method to prepare a temperature-responsive smart thermally conductive textile, which will increase the diversity of smart textiles.

\section{Declarations}

\section{Declaration of Competing Interest}

The authors declare that they have no known competing financial interests or personal relationships that could have appeared to influence the work reported in this paper.

Acknowledgments We gratefully acknowledge financial supports from the Six Talent Peaks Projects in Jiangsu Province (No. XCL-133), and the National Natural Science Foundation of China (No. 22109054).

\section{References}

1. Bai S, Xu R, Wang W, Yu D (2019) Dual-response of temperature and humidity asymmetrical cotton fabric prepared based on thiol-ene click chemistry. Colloids Surfaces A Physicochem Eng Asp 567:104-111. https://doi.org/10.1016/j.colsurfa.2019.01.050 
2. Bartkowiak G, Dabrowska A, Marszalek A (2017) Assessment of an active liquid cooling garment intended for use in a hot environment. Appl Ergon 58:182-189.

https://doi.org/10.1016/j.apergo.2016.06.009

3. Binkley HM, Beckett J, Casa DJ, et al (2002) National Athletic Trainers' Association position statement: Exertional heat illnesses. J Athl Train 37:329-343. https://doi.org/10.4085/1062-605050-9-07

4. Cao L, Emami S, Lafdi K (2014) Large-scale exfoliation of hexagonal boron nitride nanosheets in liquid phase. Mater Express 4:165-171. https://doi.org/10.1166/mex.2014.1155

5. Chen J, Huang X, Zhu Y, Jiang P (2017) Cellulose Nanofiber Supported 3D Interconnected BN Nanosheets for Epoxy Nanocomposites with Ultrahigh Thermal Management Capability. Adv Funct Mater 27:1-9. https://doi.org/10.1002/adfm.201604754

6. Chen WY, Chen LY, Ou CM, et al (2013) Synthesis of fluorescent gold nanodot-liposome hybrids for detection of phospholipase $C$ and its inhibitor. Anal Chem 85:8834-8840. https://doi.org/10.1021/ac402043t

7. Cheng D, Liu Y, Zhang Y, et al (2020) Polydopamine-assisted deposition of CuS nanoparticles on cotton fabrics for photocatalytic and photothermal conversion performance. Cellulose 27:84438455. https://doi.org/10.1007/s10570-020-03358-5

8. Couto S, Campos JBLM, Mayor TS (2011) On the performance of a mitt heating multilayer: A numerical study. Int J Cloth Sci Technol 23:373-387. https://doi.org/10.1108/09556221111166301

9. Cui Y, Gong H, Wang Y, et al (2018) A Thermally Insulating Textile Inspired by Polar Bear Hair. Adv Mater 30:1-8. https://doi.org/10.1002/adma.201706807

10. Du M, Wu Y, Hao X (2013) A facile chemical exfoliation method to obtain large size boron nitride nanosheets. CrystEngComm 15:1782-1786. https://doi.org/10.1039/c2ce26446c

11. Gao T, Yang Z, Chen C, et al (2017) Three-Dimensional Printed Thermal Regulation Textiles. ACS Nano 11:11513-11520. https://doi.org/10.1021/acsnano.7b06295

12. Guo Z, Sun C, Wang J, et al (2021) High-performance laminated fabric with enhanced photothermal conversion and Joule heating effect for personal thermal management. ACS Appl Mater Interfaces 13:8851-8862. https://doi.org/10.1021/acsami.0c23123

13. Kang W, Bi B, Zhuo R, Jiang X (2017) Photocrosslinked methacrylated carboxymethyl chitin hydrogels with tunable degradation and mechanical behavior. Carbohydr Polym 160:18-25. https://doi.org/10.1016/j.carbpol.2016.12.032

14. Li Q, Chen L, Gadinski MR, et al (2015a) Flexible higherature dielectric materials from polymer nanocomposites. Nature 523:576-579. https://doi.org/10.1038/nature14647

15. Li Q, Han K, Gadinski MR, et al (2014) High Energy and Power Density Capacitors from SolutionProcessed Ternary Ferroelectric Polymer Nanocomposites. Adv Mater 26:6244-6249. https://doi.org/10.1002/adma.201402106

16. Li Q, Zhang G, Liu F, et al (2015b) Solution-processed ferroelectric terpolymer nanocomposites with high breakdown strength and energy density utilizing boron nitride nanosheets. Energy Environ Sci 
8:922-931. https://doi.org/10.1039/c4ee02962c

17. Li Z, He Q, Ma D, Chen H (2010) On-chip integrated multi-thermo-actuated microvalves of poly(Nisopropylacrylamide) for microflow injection analysis. Anal Chim Acta 665:107-112. https://doi.org/10.1016/j.aca.2010.03.024

18. Lin Y, Connell JW (2012) Advances in 2D boron nitride nanostructures: Nanosheets, nanoribbons, nanomeshes, and hybrids with graphene. Nanoscale 4:6908-6939.

https://doi.org/10.1039/c2nr32201c

19. Lu Y, Cao J, Ren S, et al (2021) Boron nitride self-assembly cladding structure promoting thermal property and dimensional stability of polymer composites. Compos Sci Technol 201:108536. https://doi.org/10.1016/j.compscitech.2020.108536

20. Ma L, Kang H, Liu R, Huang Y (2010) Smart assembly behaviors of hydroxypropylcellulose- graftpoly(4-vinyl pyridine) copolymers in aqueous solution by thermo and $\mathrm{pH}$ stimuli. Langmuir 26:18519-18525. https://doi.org/10.1021/la103854b

21. Rodrigues Júnior JFC, Mckenna Z, Amorim FT, et al (2020) Thermoregulatory and metabolic responses to a half-marathon run in hot, humid conditions. J Therm Biol 93:102734. https://doi.org/10.1016/j.jtherbio.2020.102734

22. Salaün F, Devaux E, Bourbigot $S$, Rumeau P (2010) Thermoregulating response of cotton fabric containing microencapsulated phase change materials. Thermochim Acta 506:82-93. https://doi.org/10.1016/j.tca.2010.04.020

23. Sasidharan A, Swaroop S, Chandran P, et al (2016) Cellular and molecular mechanistic insight into the DNA-damaging potential of few-layer graphene in human primary endothelial cells. Nanomedicine Nanotechnology, Biol Med 12:1347-1355. https://doi.org/10.1016/j.nano.2016.01.014

24. Shi D, Yang M, Chang B, et al (2020) Ultrasonic-Ball Milling: A Novel Strategy to Prepare Large-Size Ultrathin 2D Materials. Small 16:1-7. https://doi.org/10.1002/smll.201906734

25. Sun W, Meng Y, Fu Q, et al (2016) High-Yield Production of Boron Nitride Nanosheets and Its Uses as a Catalyst Support for Hydrogenation of Nitroaromatics. ACS Appl Mater Interfaces 8:9881-9888. https://doi.org/10.1021/acsami.6b01008

26. Tian Z, Chen K, Sun S, et al (2019) Crystalline boron nitride nanosheets by sonication-assisted hydrothermal exfoliation. J Adv Ceram 8:72-78. https://doi.org/10.1007/s40145-018-0293-1

27. Turan E, Demirci S, Caykara T (2010) Synthesis of thermoresponsive poly(N-isopropylacrylamide) brush on silicon wafer surface via atom transfer radical polymerization. Thin Solid Films 518:59505954. https://doi.org/10.1016/j.tsf.2010.05.103

28. Wang X, Huang Z, Miao D, et al (2018) Biomimetic Fibrous Murray Membranes with Ultrafast Water Transport and Evaporation for Smart Moisture-Wicking Fabrics. ACS Nano. https://doi.org/10.1021/acsnano.8b08242

29. Wu K, Yu L, Lei C, et al (2019) Green Production of Regenerated Cellulose/Boron Nitride Nanosheet Textiles for Static and Dynamic Personal Cooling. ACS Appl Mater Interfaces 11:40685-40693. 
https://doi.org/10.1021/acsami.9b15612

30. Xia LW, Xie R, Ju XJ, et al (2013) Nano-structured smart hydrogels with rapid response and high elasticity. Nat Commun 4:1-11. https://doi.org/10.1038/ncomms3226

31. Xiao F, Naficy S, Casillas G, et al (2015) Edge-Hydroxylated Boron Nitride Nanosheets as an Effective Additive to Improve the Thermal Response of Hydrogels. Adv Mater 27:7196-7203.

https://doi.org/10.1002/adma.201502803

32. Yang A, Cai L, Zhang R, et al (2017) Thermal Management in Nanofiber-Based Face Mask. Nano Lett 17:3506-3510. https://doi.org/10.1021/acs.nanolett.7b00579

33. Yang $Y, B a o X$, Wang Q, et al (2021) Thermo-responsive cotton fabric prepared by enzyme-initiated "graft from" polymerization for moisture/thermal management. Cellulose 28:1795-1808. https://doi.org/10.1007/s10570-020-03626-4

34. Ye Y, Huang J, Wang X (2015) Fabrication of a Self-Cleaning Surface via the Thermosensitive Copolymer Brush of P(NIPAAm-PEGMA). ACS Appl Mater Interfaces 7:22128-22136. https://doi.org/10.1021/acsami.5b07336

35. You YZ, Kalebaila KK, Brock SL, Oupický D (2008) Temperature-controlled uptake and release in PNIPAM-modified porous silica nanoparticles. Chem Mater 20:3354-3359. https://doi.org/10.1021/cm703363w

36. Yu S, Chen Z, Liu J, et al (2012) Intensified cleaning of organic-fouled reverse osmosis membranes by thermo-responsive polymer (TRP). J Memb Sci 392-393:181-191. https://doi.org/10.1016/j.memsci.2011.12.025

37. Zhou B, He M, Wang P, et al (2017) Synthesis of silk fibroin-g-PAA composite using H2O2-HRP and characterization of the in situ biomimetic mineralization behavior. Mater Sci Eng C 81:291-302. https://doi.org/10.1016/j.msec.2017.08.006

38. Zhu H, Li Y, Fang Z, et al (2014) Highly Thermally Conductive Papers with Percolative Layered Boron Nitride Nanosheets. ACS Nano. 8(4),3606-3613

\section{Figures}


A

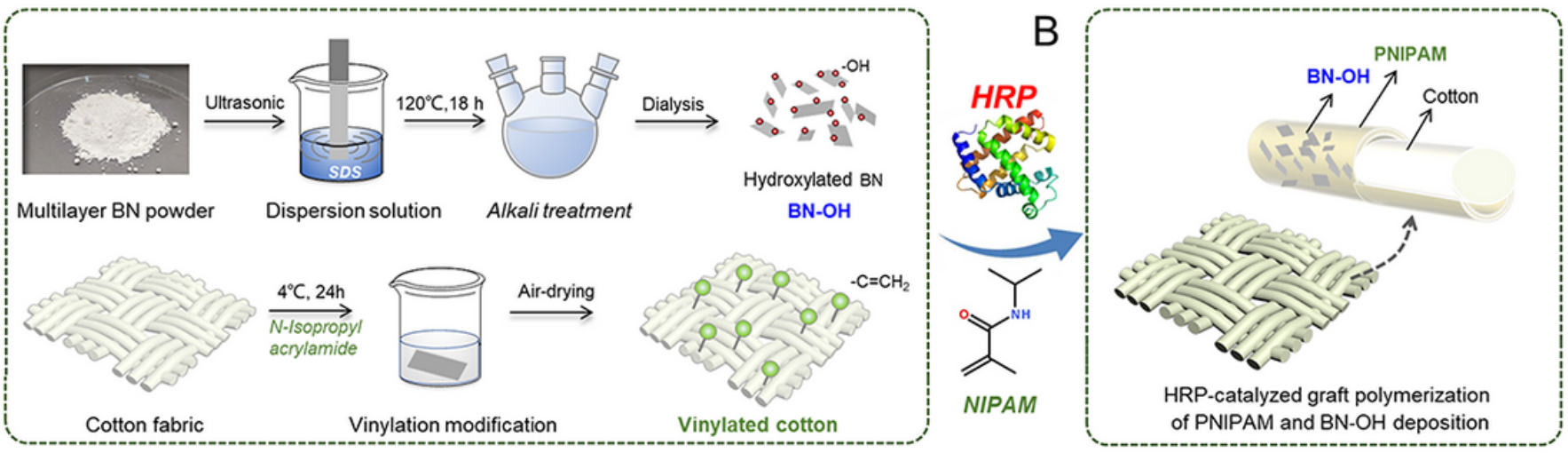

C

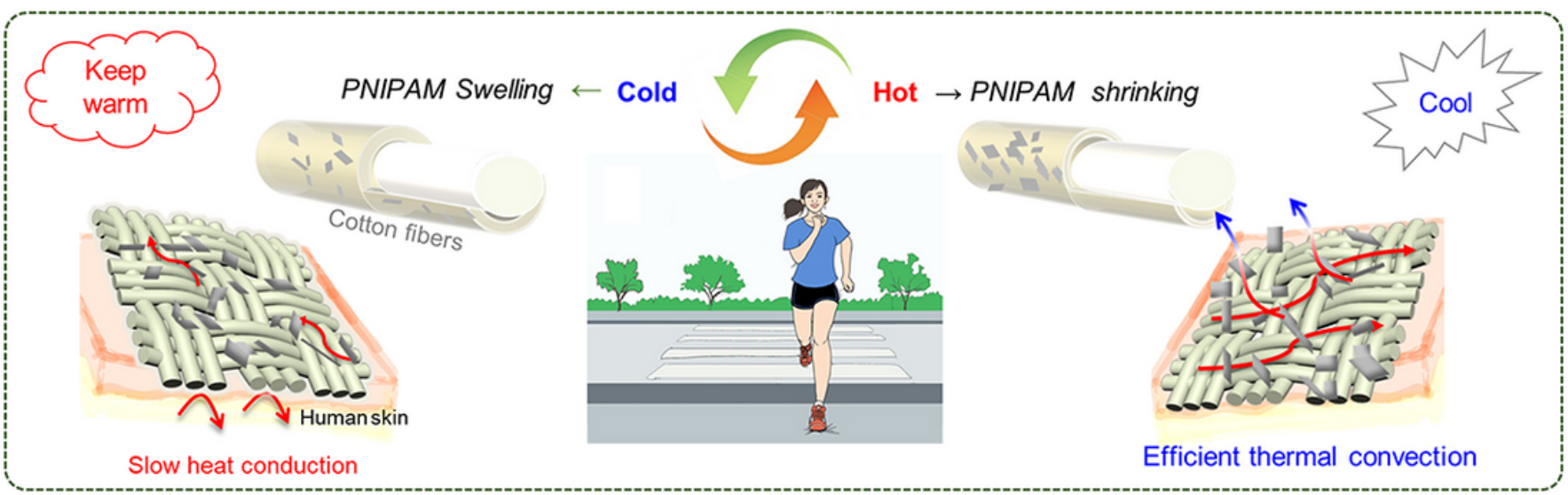

\section{Figure 1}

Schematic diagram of constructing composite cotton fabric of PNIPAM/BN-OH, preparation of BN-OH nanosheets and reactive cotton $(A)$, construction of cotton fabric with temperature response $(B)$ and the corresponding response mechanism (C) 

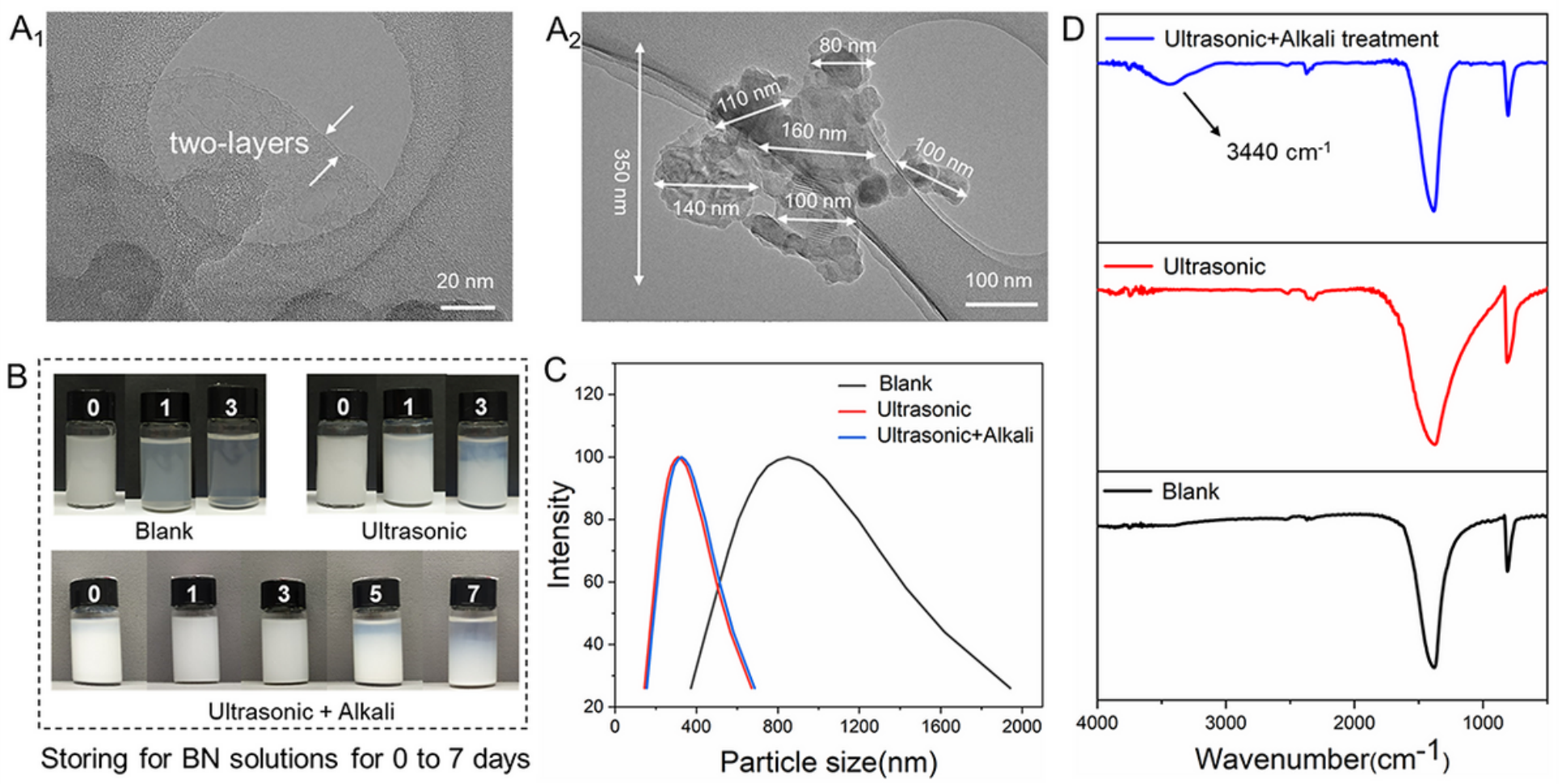

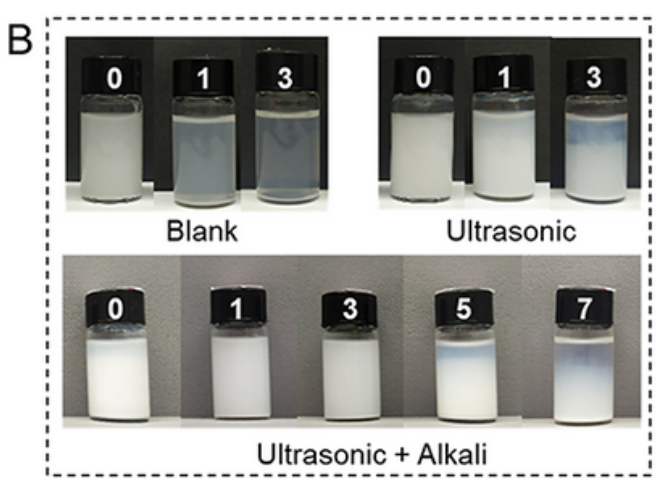

Storing for BN solutions for 0 to 7 days

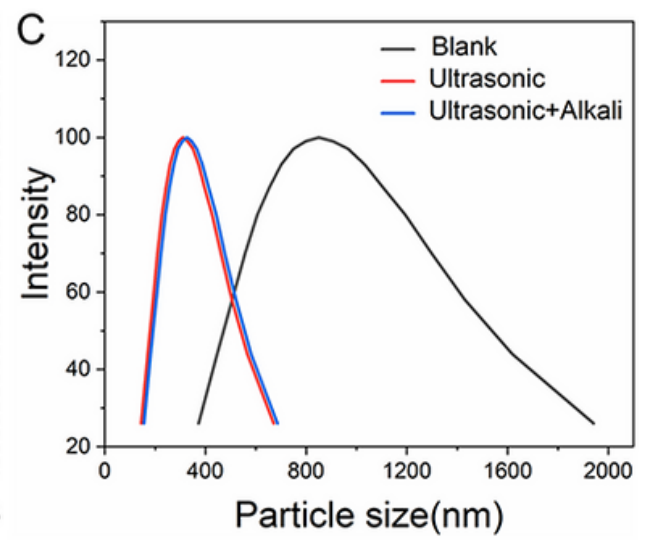

\section{Figure 2}

TEM images of $\mathrm{BN}-\mathrm{OH}$ at different magnifications (A1, and $\mathrm{A} 2)$. Suspension stability (B), particle size distribution (C), and FTIR (D) of untreated BN, ultrasonic-treated BN, and nanosheets from ultrasonicalkali treatment. 

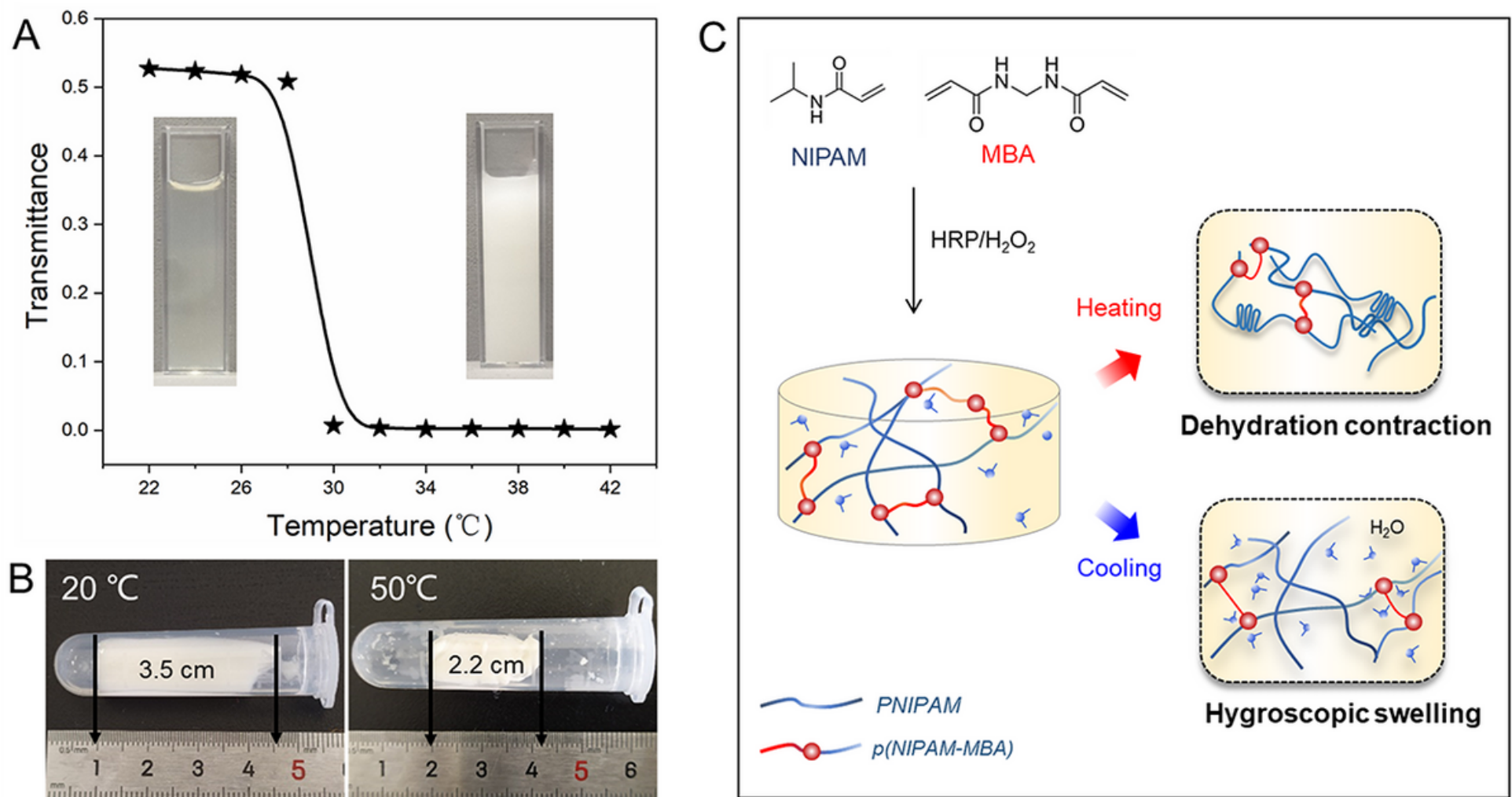

Figure 3

Transmittance of PNIPAM solutions (A), change in length of PNIPAM hydrogel at different temperatures (B), and the corresponding mechanism diagram of temperature response (C). 

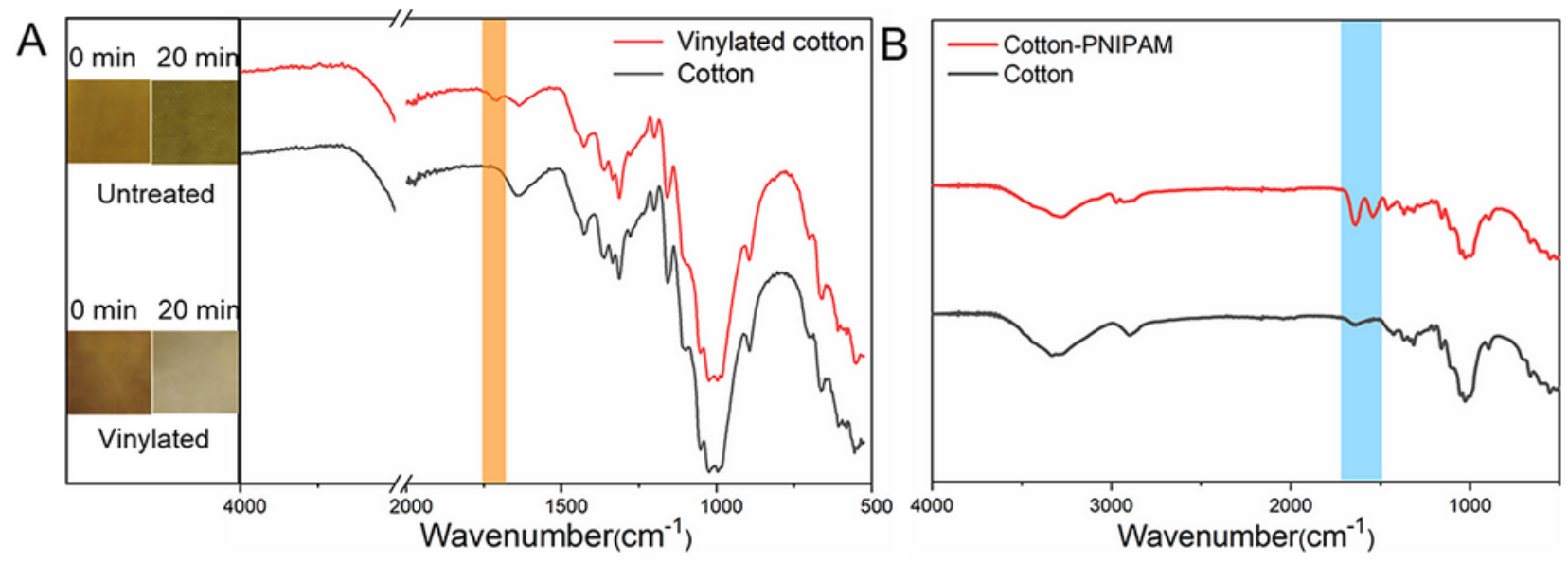

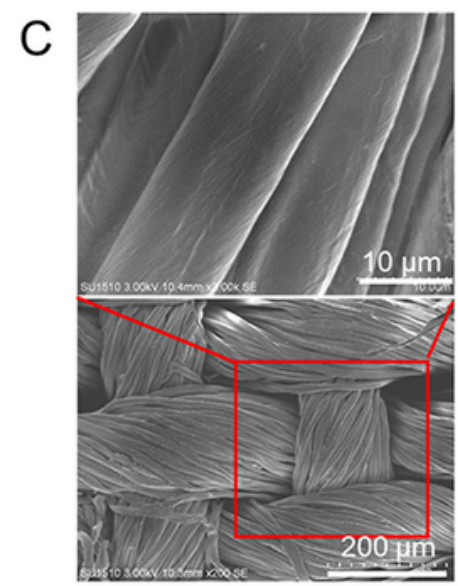

Cotton

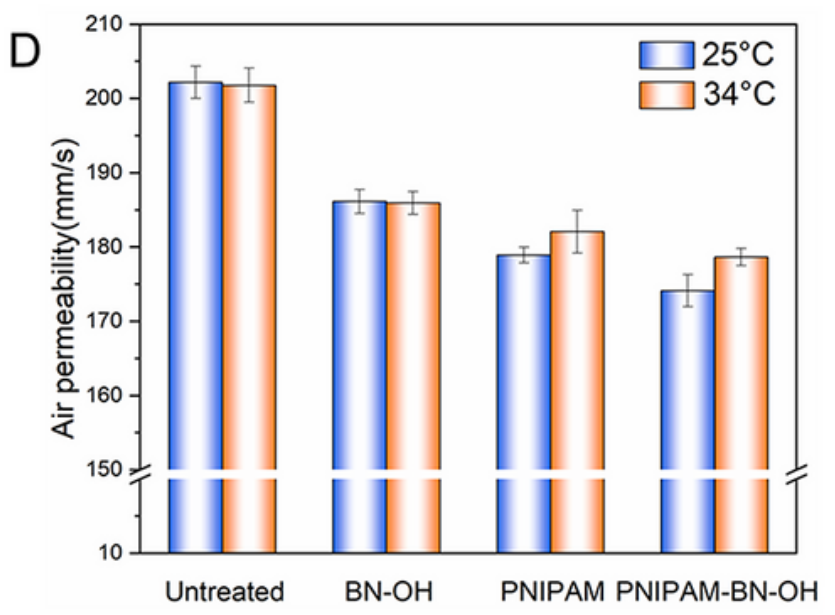

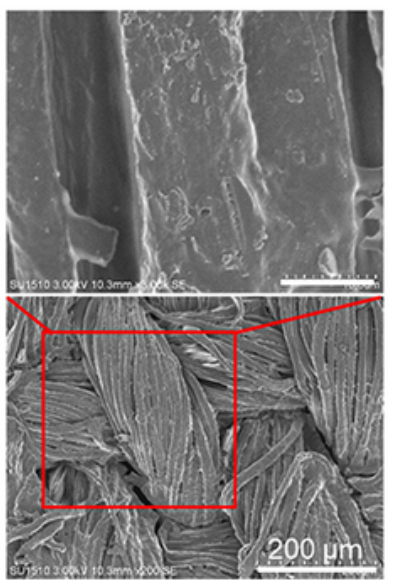

Cotton-PNIPAM

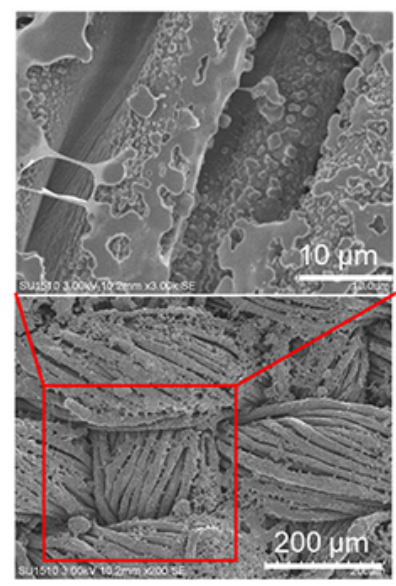

Cotton-PNIPAM-BN-OH

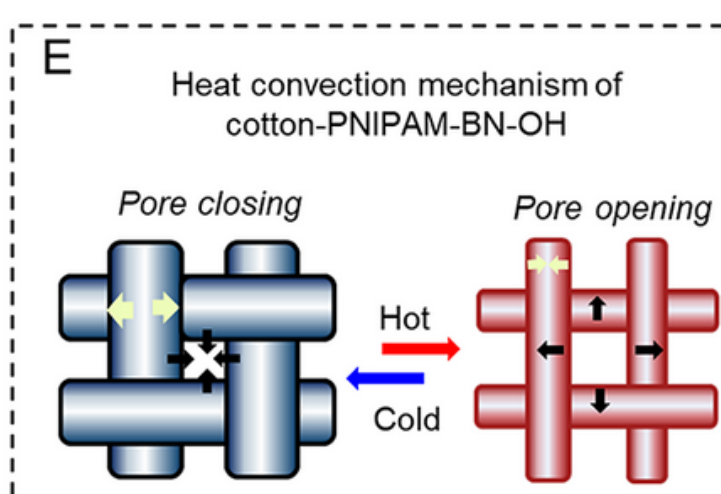

Changes in the gap sizes between yarns

\section{Figure 4}

lodine coloring and infrared spectrum of cotton (A, and B), SEM images (C), air permeability (D) of different cotton fabrics, and schematic diagram of structural changes induced by temperature response (E) 
$A_{1}$

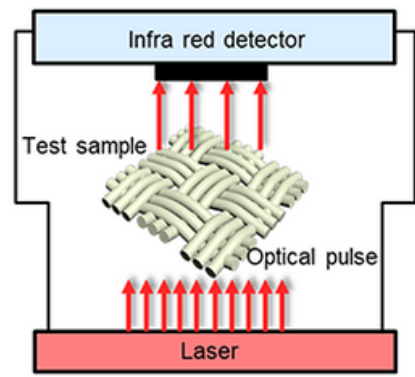

Testing principle of heat conductivity in vertical direction

$\mathrm{B}_{1}$

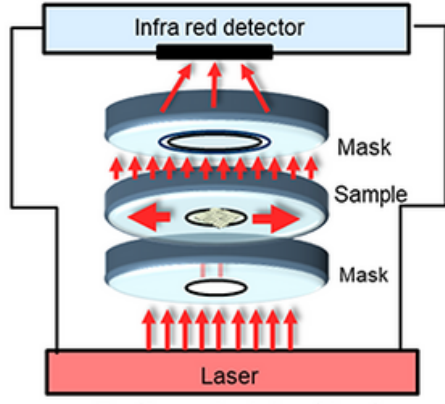

Testing principle of heat conductivity in parallel direction
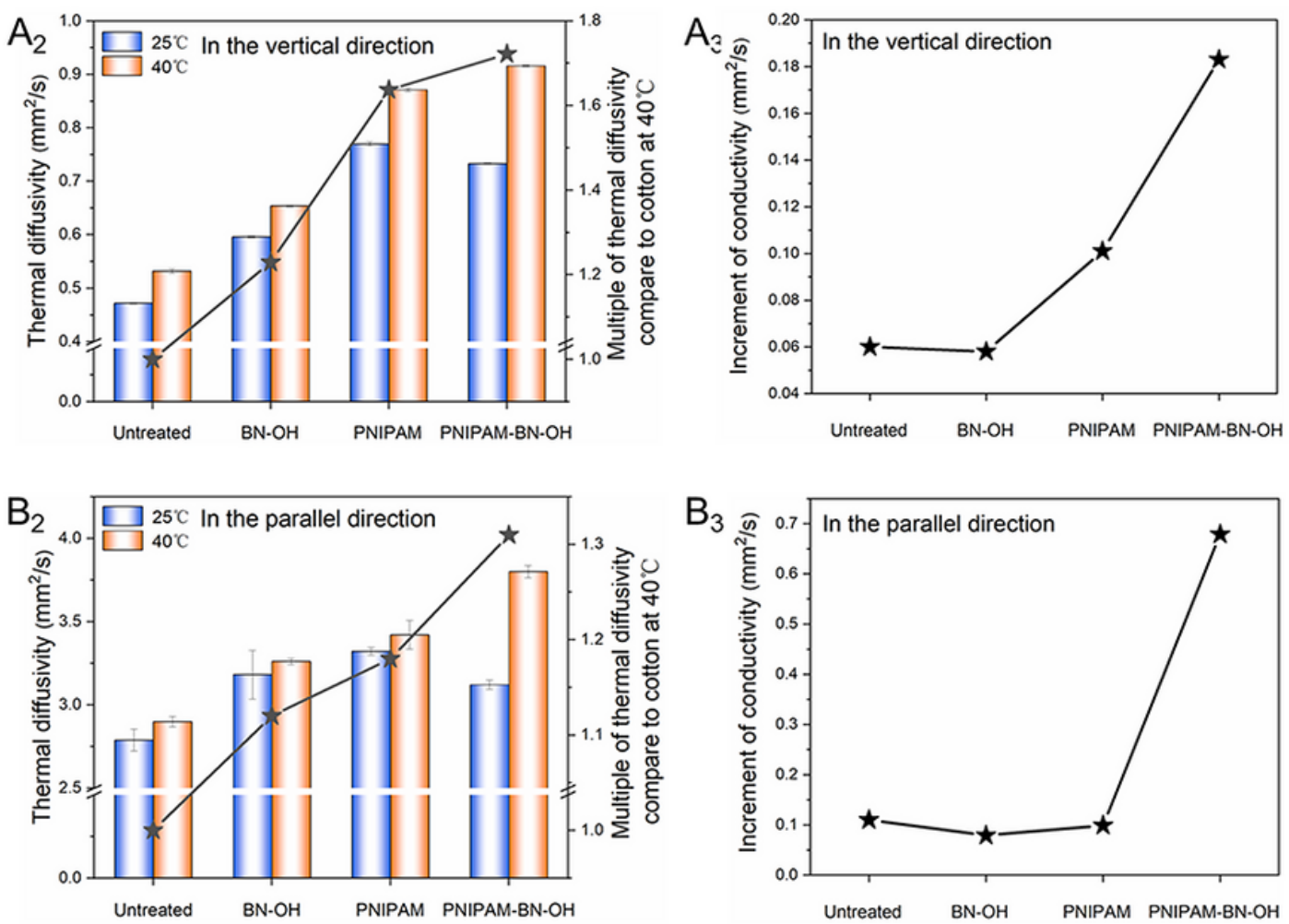

\section{Figure 5}

Schematic diagram of heat conductivity testing (A1, B1), the thermal conductivity of cotton fabrics in vertical and parallel directions (A2, B2), and the increments of thermal conductivity under different temperatures $(\mathrm{A} 3, \mathrm{~B} 3)$. 

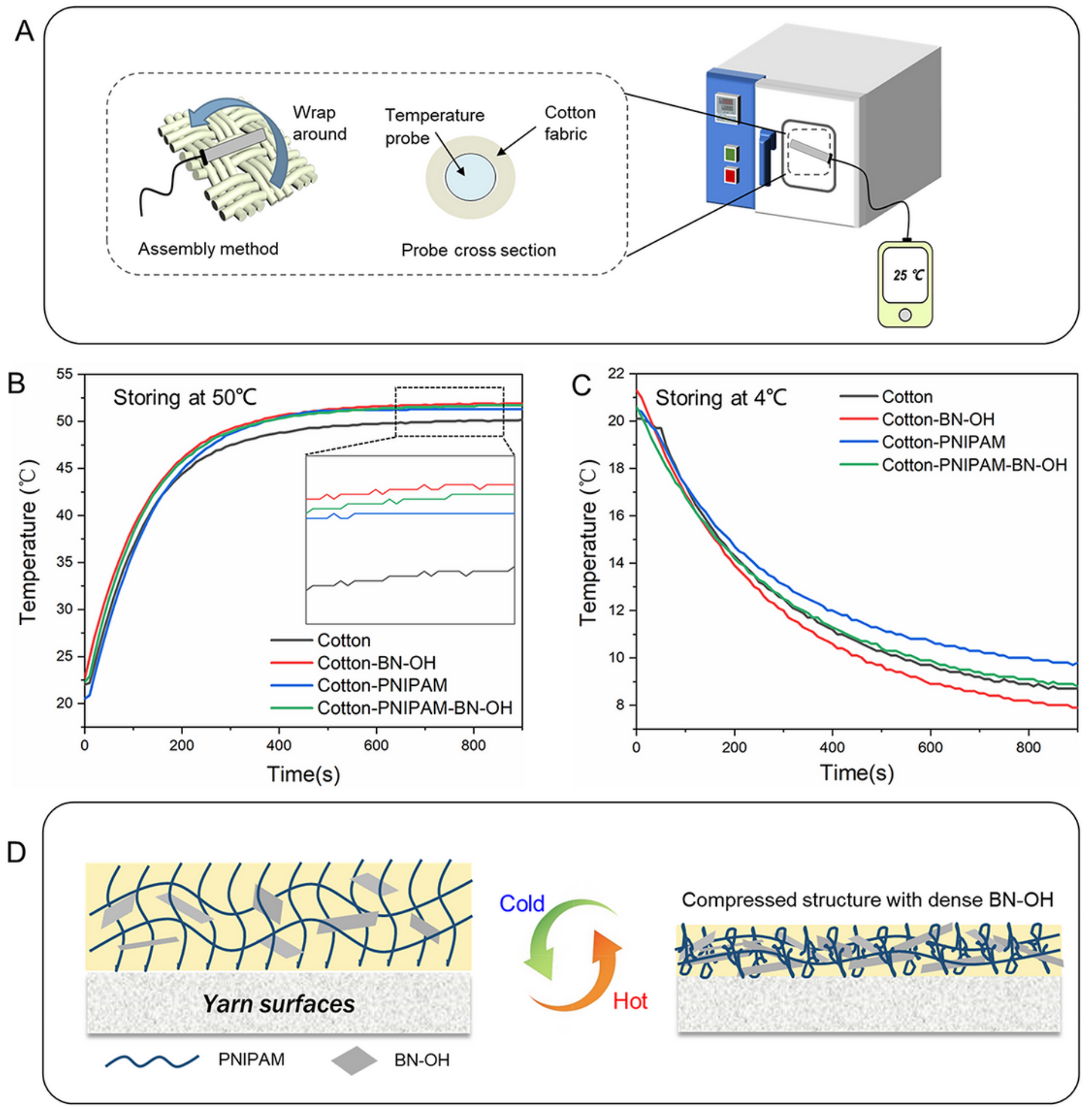

\section{Figure 6}

A device for determining dynamic temperature changes $(A)$, the detected temperature inside the different warped fabric sample at $55^{\circ} \mathrm{C}$ and $4^{\circ} \mathrm{C}$, respectively $(\mathrm{B}, \mathrm{C})$, and schematic diagram of temperature response for the composite fabric (D). 
Figure 7

Infrared thermal images of fabrics on hand skin before and after $10 \mathrm{~min}$ of sporting $(\mathrm{A})$, the average surface temperature before (B), and after sporting (C).
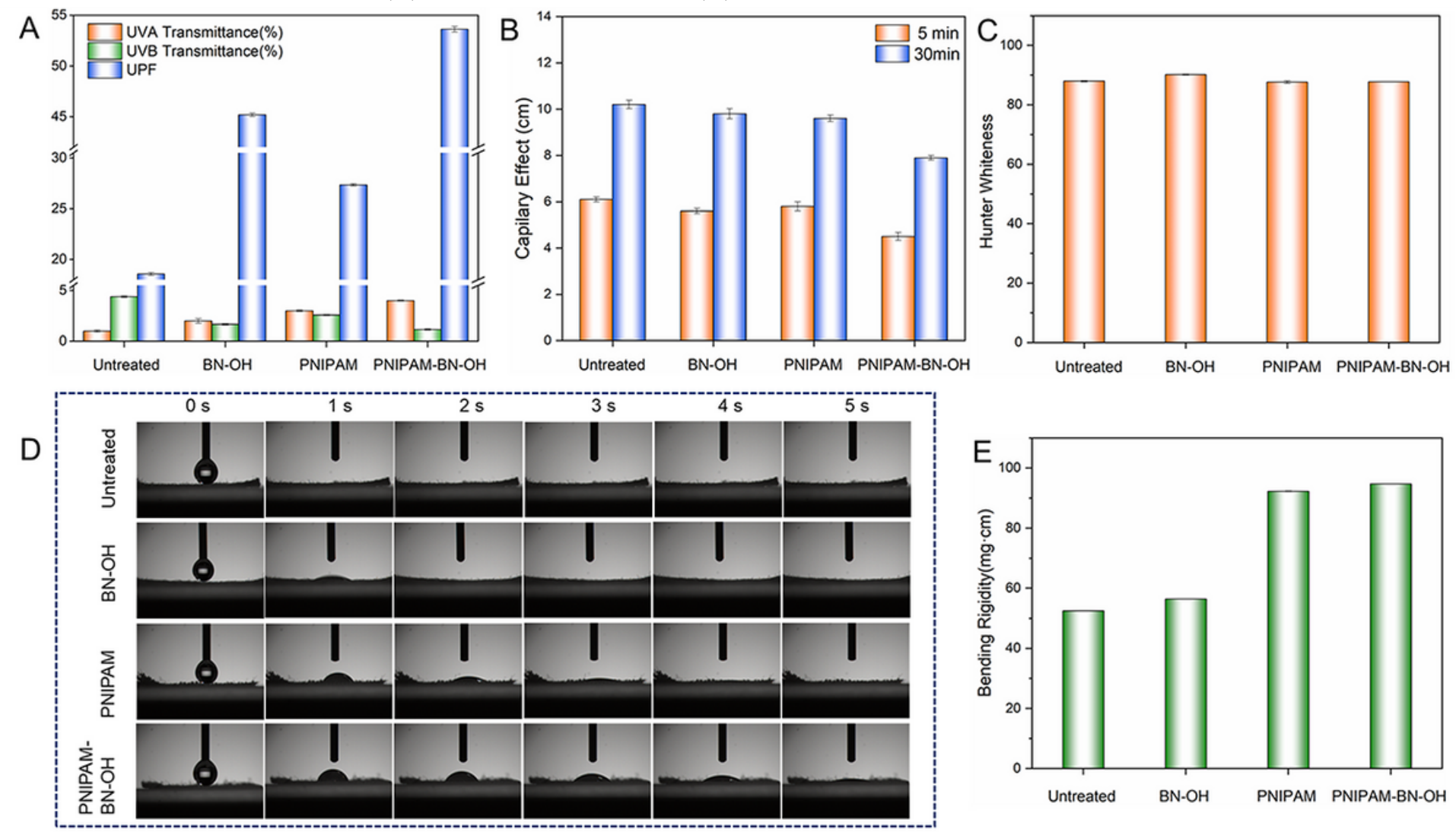

Figure 8

UV resistance ability (A), Hunter whiteness (B), wetting height (C), water contact angle images (D), and bending rigifity $(\mathrm{E})$ of the cotton fabrics based on different treatments.

\section{Supplementary Files}

This is a list of supplementary files associated with this preprint. Click to download.

- graphicalabstract.png

- SupportingInformation.docx 